\title{
Application of Temperature Modulation-SDP on MOS Gas Sensors: Capturing Soil Gaseous Profile for Discrimination of Soil under Different Nutrient Addition
}

\author{
Arief Sudarmaji ${ }^{1,2}$ and Akio Kitagawa ${ }^{1}$ \\ ${ }^{1}$ Electrical Engineering and Computer Science, Kanazawa University, Kakuma-machi, Kanazawa, Ishikawa 920-1192, Japan \\ ${ }^{2}$ Agricultural Engineering, University of Jenderal Soedirman, Jalan Dr. Soeparno, Karangwangkal, Purwokerto, \\ Central Java 53123, Indonesia
}

Correspondence should be addressed to Arief Sudarmaji; arief.unsoed@gmail.com

Received 24 January 2016; Accepted 28 February 2016

Academic Editor: Kourosh Kalantar-Zadeh

Copyright ( 2016 A. Sudarmaji and A. Kitagawa. This is an open access article distributed under the Creative Commons Attribution License, which permits unrestricted use, distribution, and reproduction in any medium, provided the original work is properly cited.

\begin{abstract}
A technique of temperature modulation-SDP (specified detection point) on MOS gas sensors was designed and tested on their sensing performance to such complex mixture, soil gaseous compound. And a self-made e-nose was built to capture and analyze the gaseous profile from sampling headspace of two soils (sandy loam and sand) with the addition of nutrient at different dose (without, normal, and high addition). It comprises (a) $6 \mathrm{MOS}$ gas sensors which were driven wirelessly on a certain modulation through (b) a PSoC CY8C28445-24PVXI-based interface and (c) the Principal Component Analysis (PCA) and neural network $(\mathrm{NN})$ as pattern recognition tools. The gaseous compounds are accumulated in a static headspace with thermostatting and stirring under controlled condition to optimize equilibration and gases concentration as well. The patterns are trained by backpropagation algorithm which employs a log-sigmoid function and updates the weights using search-then-converge schedule. PCA results indicate that the sensor array used is able to differentiate the soil type clearly and may provide a discrimination as a response to presence/level of the nutrients addition in soil. Additionally, the PCA enhances the classification performance of NN to discriminate among the predescribed nutrient additions.
\end{abstract}

\section{Introduction}

One promising technique that may overcome the crossselectivity problem on MOS gas sensor and also increase its sensitivity is temperature modulation [1]. It alters the kinetics of the sensor due to a cyclic variation of operational temperature resulting from the changes of the working voltage periodically. The sensor temperature is determined by the amplitude of the voltage applied across its heater. The cyclic kinetics of MOS gas sensor will lead to different rates of reaction which seem to follow the given modulation, so as to provide a unique response for each gas dependent on either shape or amplitude of modulation [1-3]. The operating modulation voltage may change periodically by either square/rectangular or sine waveform or triangular/saw-tooth [3].
Temperature modulation has successfully enhanced the selectivity of (thick/thin) $\mathrm{SnO}_{2}$ in distinguishing a single or multicomponent by applying certain modulation or analysis for optimum result as reported in [4-6], even on catalytic gas sensor [7]. Moreover, it also succeeded on the TGSs [8-11]. In the early investigation of this technique, pulse/thermalcycling technique was used which mostly was applied on TGSs as reported by Lee and Reedy [1]. Most of investigations utilized the modulation generator and digital acquisition/ signal recorder separately. It is difficult to take the advantages of temperature modulation for field in situ measurement.

We introduced a technique, namely, the temperature modulation with specified detection point (temperature modulation-SDP) (see Section 2), which is able be applied to drive a single/array of MOS gas sensors [12]. By applying 


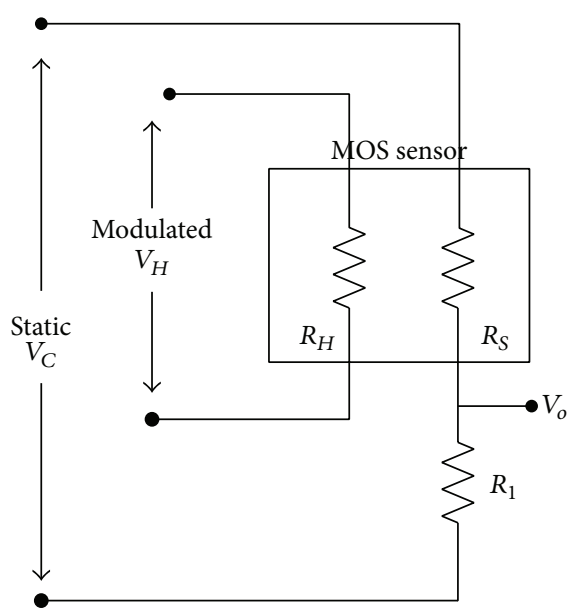

(a)

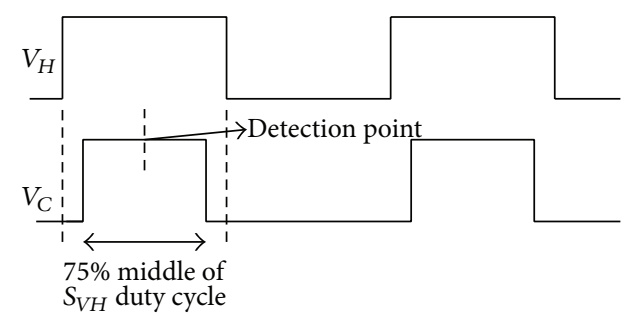

(c)

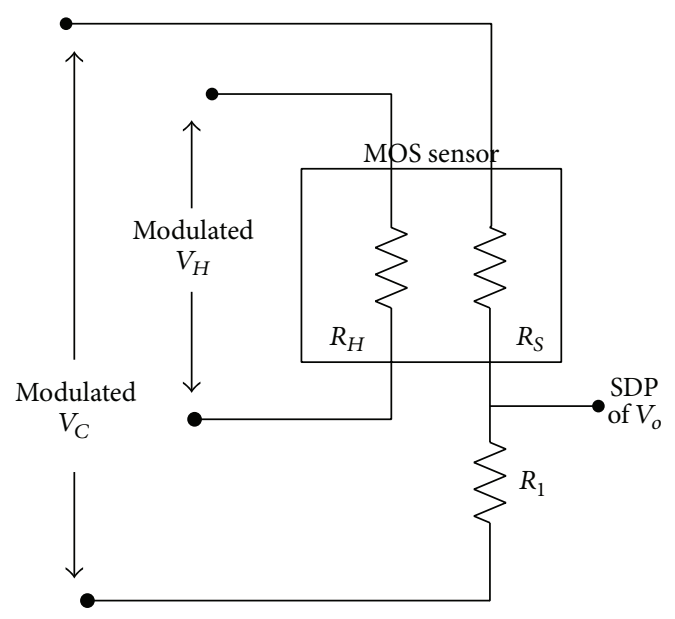

(b)

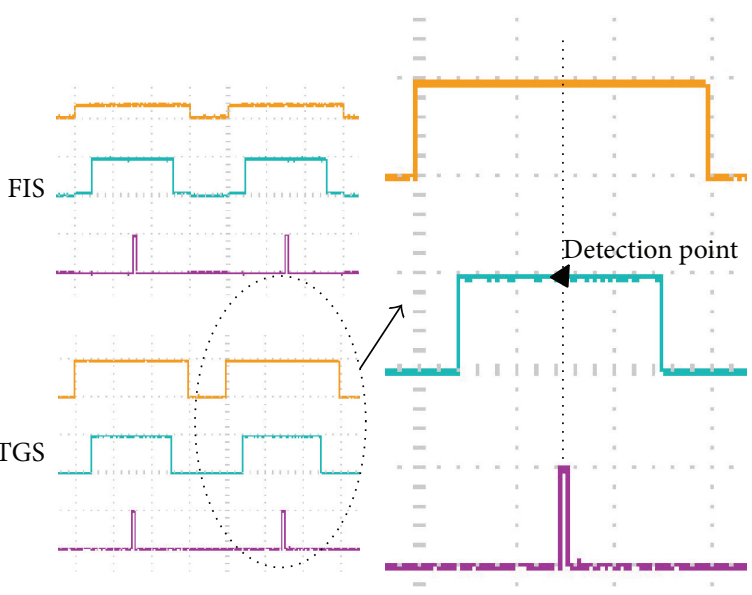

(d)

FIGURE 1: Principle and basic schematic of (a) general temperature modulation and (b) temperature modulation-SDP with (c) the desire rectangular modulation and $(\mathrm{d})$ the generated waveform modulation.

rectangular temperature modulation-SDP, we tested 6 MOS gas sensors (3 TGSs and 3 FISs) in a PSoC-based e-nose system where the result shows that there is a significant increment of selectivity in discriminating 3 single volatile compounds (toluene, ethanol, and ammonia) compared with static temperature operation [12].

Since those 6 MOS gas sensors (TGS2444, TGS2602, TGS825, FISAQ1, FISSB30, and FIS12A) are designed by their manufacturers to sense the volatile compounds, to further test for the sensitivity and selectivity, we aim to assess the temperature modulation-SDP in distinguishing a such complex compound in variable conditions. We therefore tested the performance of the rectangular temperature modulationSDP for evaluating the influence of soil type and nutrient addition on their responses. Soils, a complex mixture, are composed mostly of minerals and organic materials, water, air, and countless organisms [13, 14]. Many gases, mostly volatile organic compounds, are found at soil atmosphere due to microbial activity in which the type and the concentrations of VOCs produced may differ because of differences in community composition or nutrient availability [15-17]. Soil also is known to have a unique smell that can be sensed with human olfaction system, resulting from two special molecules (geosmin and methylisoborneol) due to the activity of bacteria, mostly belonging to the genus Streptomyces $[18,19]$. We tested two soils (sandy loam and sand) with the following addition of commercial compost in different dose (without, normal, and high).

\section{The Temperature Modulation with Specified Detection Point (SDP)}

In principle, the temperature modulation-SDP is similar to general temperature modulation (Figure 1(a)), yet, besides a modulation on heater unit $\left(V_{H}\right)$, it also modulates the sensing unit $\left(V_{C}\right)$ concurrently and in the same phase with $V_{H}$ (Figure $1(\mathrm{~b})$ ). The $V_{C}$ is positioned on midpoint $75 \%$ of "on/high" state of $V_{H}$ (Figure 1(c)). The SDP means that the time of output detection of MOS gas sensor is put at specified point. The SDP ensures the same measurement point at each output shape. Moreover, the $V_{C}$ which is associated with $V_{H}$ may lead to prevention of the sensor from possible migration 
TABLE 1: MOS gas sensors used and typical gas target claimed.

\begin{tabular}{lccc}
\hline Number & Sensor & Gas target & Working range \\
\hline 1 & TGS2444 & Ammonia & $1-100 \mathrm{ppm} \mathrm{[20]}$ \\
2 & TGS2602 & Air contaminant & $1-30 \mathrm{ppm} \mathrm{of} \mathrm{EtOH} \mathrm{[44]}$ \\
3 & TGS825 & Hydrogen sulfide & $5-100 \mathrm{ppm} \mathrm{[36]}$ \\
4 & FIS12A & Methane & $300-7,000 \mathrm{ppm} \mathrm{[45]}$ \\
5 & FIS30SB & Alcohol & $1-100 \mathrm{ppm} \mathrm{[46]}$ \\
6 & FISAQ1 & VOC (air quality) & $10-10,000 \mathrm{ppm} \mathrm{[47]}$ \\
\hline
\end{tabular}

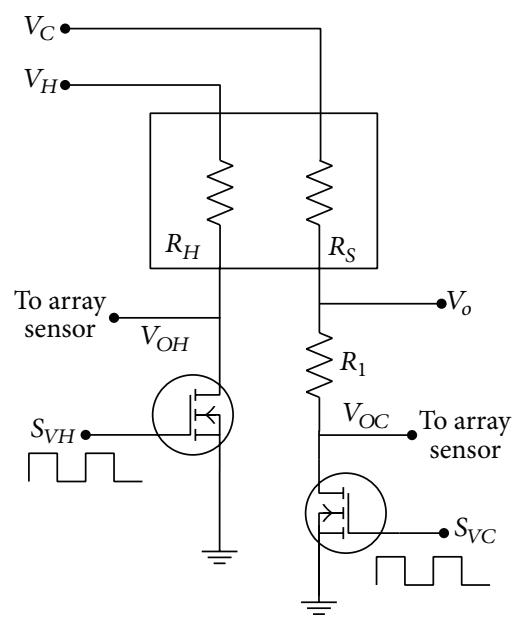

(a)

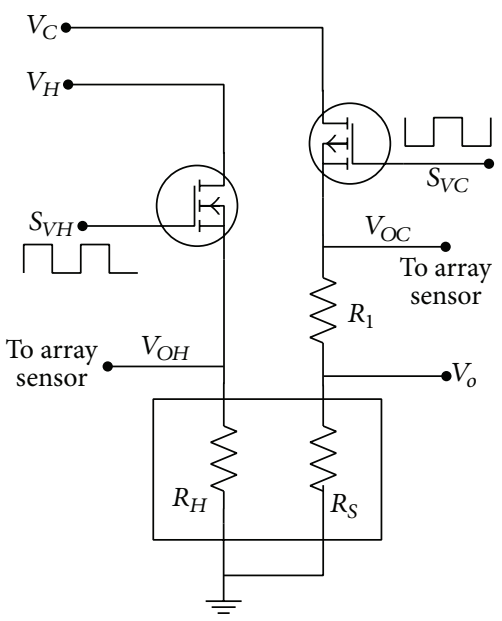

(b)

FIGURE 2: The schematic of temperature modulation-SDP for array TGSs (a) and FISs (b). $V_{H}$ and $V_{C}$ are static voltage, $V_{C}$ is sensing circuit voltage, $S_{V H}$ is modulation signal for $V_{H}$, and $S_{V C}$ is modulation signal for $V_{C}$.

of heater materials into the sensing material which could cause long term drift of sensing material's resistance to higher values [20].

This technique allows a single chip (such as controller, processor, or hybrid) to get the advantages of temperature modulation by concomitantly generating modulation signal and acquiring the output at a constant point as well in the chip itself, even when using many MOS gas sensors. Generally a handheld device employs a single chip processor or controller as the heart of system and its time consumption depends on the clock used and complexity of tasks and features involved (sequential multiplexing, digital/analog conversion, I/O handling, timer interruption, communicating with outer device, etc.). Lower-end chip will spend more time. However, the flexibility to be set as a custom-developed system could actually be an advantage in an application, like a sensors handling [21, 22].

In this study, we put on a rectangular modulation and a single detection point at middle of modulation of sensing unit as shown in Figure 1(c). Figure 1(d) shows that the waveforms modulation (captured by Oscilloscope Tektronix TDS 2024B) at heater unit (yellow) and sensing unit (green) and the zone of detection point of overall MOS gas sensors used (purple) meet the desired modulation in Figure 1(c).

We designed the schematic of single temperature modulation-SDP for each array of TGSs and FISs, respectively, as shown in Figure 2, since there is slight difference in configuration on them. It employs common modulation circuits employing FET (Field Effect Transistor). In particular, on TGS244, we constructed an individual modulation circuit because it requires a recommended modulation as noted in its datasheet [20]. Both TGSs and FISs are configured in voltage divider as standard technique for measuring resistance changes [23].

\section{The Self-Made E-Nose}

We built a PSoC-based e-nose that consists of 3 main units: (1) sensing unit, 6 MOS gas sensors (Table 1), which are expected to sense the soil volatile compounds since they are specified to detect a particular volatile compounds in low concentration range, and 2 environment sensors (LM35 and HSM30G) to monitor temperature and humidity in chamber; (2) a PSoC CY8C28445-24PVXI-based interface system; and (3) PCA and NN as preprocessing and pattern recognition, respectively.

As shown in Figure 3, a single CY8C28445-24PVXI used acts as a core of system which mainly functioned to generate desired modulation signals, to acquire all sensors output, and to communicate with computer wirelessly. It connects through radiofrequency using XBee (IEEE 802.15.4) serial communication interfaced by a developed program under 


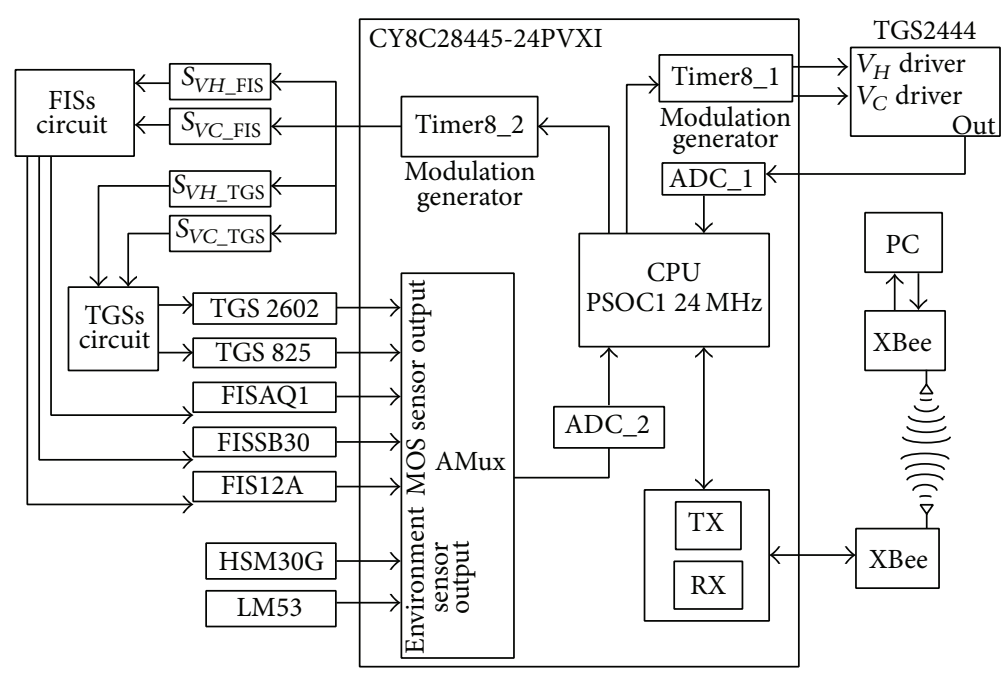

FIGURE 3: Block diagram of PSoC-based e-nose system for capturing soil gaseous profile.

Visual Basic.Net 2012. We configured some analog blocks (PGAs, Multiplexer, ADCs, and Switched Capacitor) and digital (Timer8, Counter and PWM for ADC, and UART) blocks inside the PSoC to comply with the functions. For more detailed diagram and configuration of the PSoC, refer to our previous works $[12,24]$. The PSoC firmware was built using PSoC Designer 5.4.

We developed the software of PCA and NN using Visual Studio 2012 to analyze the profiles of the array sensor responses corresponding to the soil samples. The PCA software is constructed by utilizing PCA routine in opensource Accord.NET Framework 2.10. The NN was developed based on backpropagation (BP) learning method in Multilayer Perceptron Neural Network (MLPNN) architecture by employing a log-sigmoid activation function. The weights are updated using global adapted learning parameter $\eta$ updated by search-then-converge schedule. It is a simple and nonadaptive annealing schedule. Typically, it starts with large $\eta$ and gradually decreases as the learning proceeds in which the process of adapting $\eta$ is similar to that in simulated annealing [25]. Basically the BP algorithm is a generalization of the delta rule (Least-Mean Squares algorithm), also called the generalized delta rule, which uses a gradient search technique to minimize a cost function equivalent to the Mean Square Error (MSE) between actual network outputs and the desired (target) output [25]. The BP propagates the MSE backward through the network and the weights (and biases) are then adjusted by a gradient descent based algorithm. Thus, a closed-loop control system is established in network.

\section{Material and Method}

4.1. Soil Preparation and Sample Handling. The sandy loam and sand soil were derived from the top $15 \mathrm{~cm}$ and land without prior soil management. Sandy clay loam soil was taken from land around Kanazawa University $\left(36^{\circ} 32^{\prime} 46.3380^{\prime \prime} \mathrm{N}\right.$, $136^{\circ} 42^{\prime} 11.5452^{\prime \prime} \mathrm{E}$ ), while sand soil was taken from around coastal area of Uchinada Beach $\left(36^{\circ} 38^{\prime} 39.19^{\prime \prime} \mathrm{N}\right.$, $\left.136^{\circ} 37^{\prime} 37.88^{\prime \prime} \mathrm{E}\right)$, a sand hill on Sea of Japan, which is located about $17 \mathrm{~km}$ from Kanazawa University. The collected soil samples were crushed and sieved manually at $<2 \mathrm{~mm}$ after plant derbies, turfs, and gravels were carefully removed. As soil treatments, we added an amount of fermentation compost. The compost is given at average/normal and high doses as recommended in practical application, that is, 20 and 30 tons ha ${ }^{-1}$ DM (Dry Matter), respectively [26]. Taking into account a general assumption that, in 1 ha soil area, $15 \mathrm{~cm}$ deep, that contains $2 \mathrm{Mkg}$ despite bulk density of soil varying considerably [27], we therefore added the compost at 0,15 , and $22.5 \mathrm{mg} / \mathrm{g}$ soil sample which approached doses of 0,20 , and 30 tons ha $^{-1} \mathrm{DM}$, respectively.

The soil and compost samples were put into LLDPE (linear low-density polyethylene) plastic bag and sealed with paraffin. Then, we stored them in refrigerator at $5 \pm 0.5^{\circ} \mathrm{C}$ to inactivate microbial activity in soil. This temperature is known as biologic zero temperature, which recognized that most microbes in soil become relatively inactive at temperature below $5^{\circ} \mathrm{C}[28,29]$. Prior to being used, the samples were air-dried up to room temperature.

We prepared the samples into solution since soil contains many soluble substances in water and liquid has bigger diffusion coefficient than solid and thus leads to shorter diffusion times. We calculate the mass of soil sample using (1) to obtain the mass of pure water and compost addition, where $m_{s}$ expresses mass of soil $(g), V_{v}$ is volume of headspace vial $(\mathrm{mL}), \rho_{s}$ is bulk density of soil (sandy loam $=1.44 \mathrm{~g} / \mathrm{mL}$ and sand $=1.52 \mathrm{~g} / \mathrm{mL}[30,31]), \rho_{w}$ is density of pure water $=0.998 \mathrm{~g} / \mathrm{mL}, \beta\left(V_{G} / V_{S}\right)$ is phase ratio in $\mathrm{SH}$, and $w_{c}$ is water content (in fractional number). Table 2 resumes the properties of parameters used and calculation results:

$$
m_{s}=\frac{V_{v} \times \rho_{s} \times \rho_{w}}{(\beta+1) \times\left(\rho_{w}+w_{c} \times \rho_{s}\right)} .
$$

4.2. Measurement Procedures. The soil gaseous compounds are accumulated in a static headspace $(\mathrm{SH})$ and the headspace 
TABle 2: Properties of samples of soil, fertilizer, water, and static headspace condition.

\begin{tabular}{lc}
\hline Properties of SH & Value \\
\hline Volume of SH vial & $90 \mathrm{~mL}$ \\
Bulk density of sandy loam soil & $1.44 \mathrm{~g} / \mathrm{mL}$ \\
Bulk density of sand soil & $1.52 \mathrm{~g} / \mathrm{mL}$ \\
Phase ratio & 1.5 \\
Water content & $100 \%$ \\
Density of pure water & $0.998 \mathrm{~g} / \mathrm{mL}$ \\
Calculation results & \\
Mass of sandy loam soil & $21.22 \mathrm{~g}$ \\
(i) Mass of compost adding at 20 ton/ha & $0.318 \mathrm{~g}$ \\
(ii) Mass of compost adding at 30 ton/ha & $0.477 \mathrm{~g}$ \\
Mass of sand soil & $21.63 \mathrm{~g}$ \\
(i) Mass of compost adding at 20 ton/ha & $0.324 \mathrm{~g}$ \\
(ii) Mass of compost adding at 30 ton/ha & $0.287 \mathrm{~g}$ \\
\hline
\end{tabular}

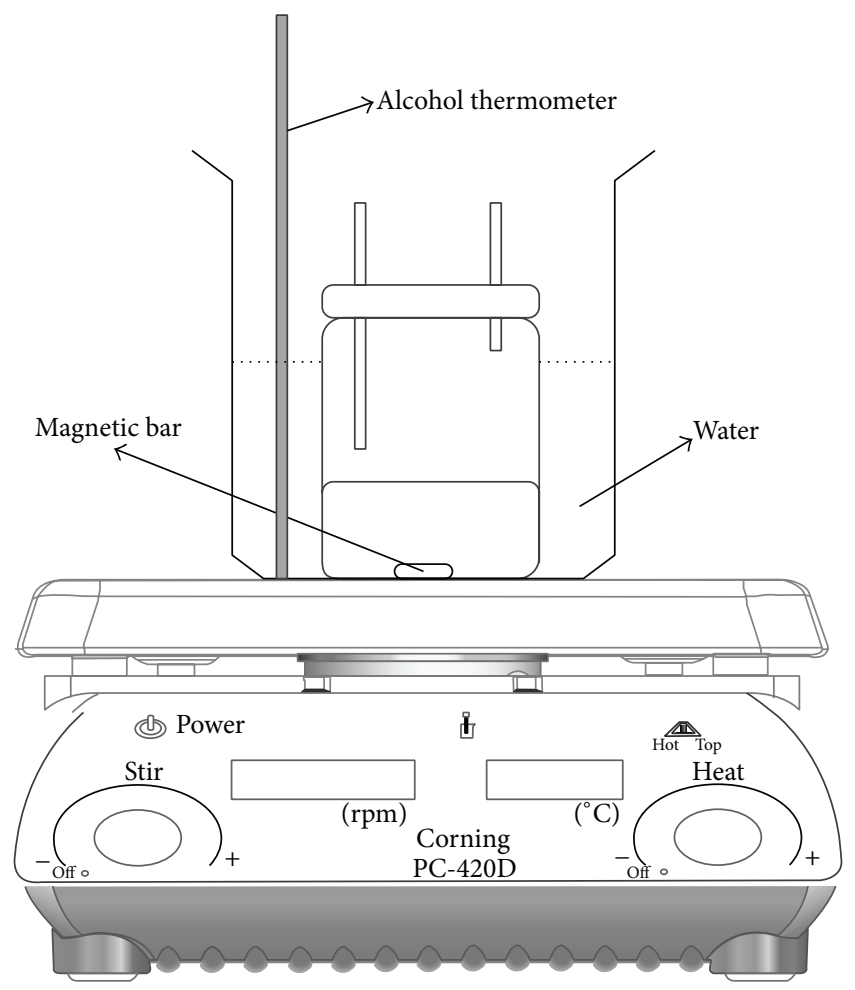

FIGURE 4: Headspace conditioning with heating and stirring using Corning PC-420D in SH sampling, the layout of Corning modified from [32].

equilibration is optimized by both agitating (i.e., stirring) and thermostatting concurrently for all samples on the same phase ratio. We set 30 minutes, $60^{\circ} \mathrm{C}$, and $200 \mathrm{rpm}$ of equilibration time, temperature, and stirring frequency, respectively. We utilized Corning PC-4200D to heat and stir the sample in the $\mathrm{SH}$ vial. We used $90 \mathrm{~mL}$ glass container with sealed cap as headspace vial which is put inside the $500 \mathrm{~mL}$ open beaker filled with $100 \mathrm{~mL}$ water (Figure 4). It aims to maintain the equilibrium relative humidity the same as the soil sample. And the headspacing was conducted inside a room under controlled temperature. By those ways, all soil samples were under the same treatments and environmental conditions.

The temperature modulation is set on $0.25 \mathrm{~Hz}, 75 \%$ duty cycle to drive all MOS gas sensors, except for TGS2444 [20], which is on its recommended duty cycle. The initial action of the MOS gas sensors, after a long inactive state, is carried out for one hour of measuring the reference gas to allow them to reach a stable condition. The gas sensors are expressed in resistance and the profile is defined by its sensitivity $(S)$ [4], where $R_{0}$ is sensor resistance of air and $R_{g}$ is sensor resistance of soil gaseous compound (see (2)):

$$
S=\frac{R_{0}}{R_{g}} .
$$

The measurement of soil gaseous profiles is performed using close measurement method by switching between the reference gas (filtered air with silica gel) as baseline and analyte gas (soil gaseous compounds). The flow direction and rate of gas are controlled by 3-way valve and the Kofloc mass flow controller (MFC), respectively. The MFC are set at $0.3 \mathrm{lpm}$. As shown in Figure 5, the reference gas flows through point (a) (valve-1), point (c) (valve-2), and point (e) (valve-3), while the analyte gas flows through point (b) (valve-1), point (d) (valve-2), and point (e) (valve-3). The purging of sensor chamber was in open measurement mode by disconnecting the hose of inlet pump from valve-2, directing valve-3 to point (f), and turning on the purge pump.

At preresearch, we observed $R_{g}$ for 5 minutes after $R_{0}$ measurement to determine the response of each sensor and obtain the best starting measurement time for $R_{g}$ measurement. Significantly, we found that overall sensors reached a stable state after $\pm 150 \mathrm{~s}( \pm 2.5 \mathrm{~min})$ which strongly indicate that they are sensing stably the flow of gas that have been spread evenly in the close measurement system. We therefore took this time to be the starting point of $R_{g}$ measurement. Thus, we set the total measurement time per sample as 37 minutes, covering the phases of the headspace (30 minutes), $R_{0}$ measurement (1 minute), stabling time (2.5 minutes), $R_{g}$ measurement (1 minute), and purging (5 minutes) sequentially. The sampling period of both $R_{0}$ and $R_{g}$ measurement was 2 seconds, and their averages were used to represent the baseline and soil gaseous compound.

\section{Results and Discussion}

5.1. Individual Sensitivity-Based Response of MOS Gas Sensors. Individual sensitivity-based soil gaseous profiles of MOS gas sensors used on each soil type with the different dose of nutrient addition are shown in Figure 6. It reveals that the array of gas sensors was able to sense the soil gases and/or volatile compounds resulting from different samples and as well indicates that the method of the optimized SH seems suitable for providing/accumulating the concentration sufficiently. Those individual responses indicate that the technique of temperature modulation-SDP led the sensors to sense differently 


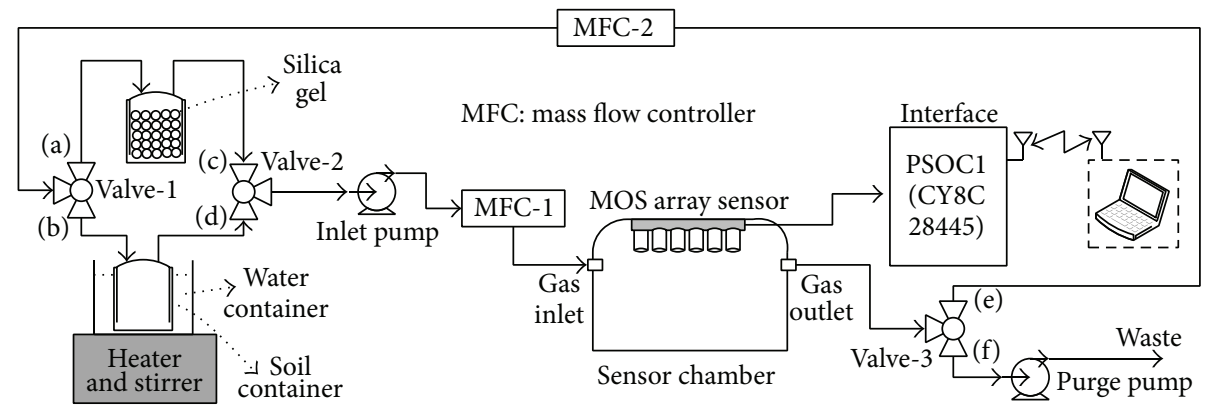

Figure 5: Experimental setup to capture the soil gaseous compounds using static headspace extraction in sample flow system (close) measurement.

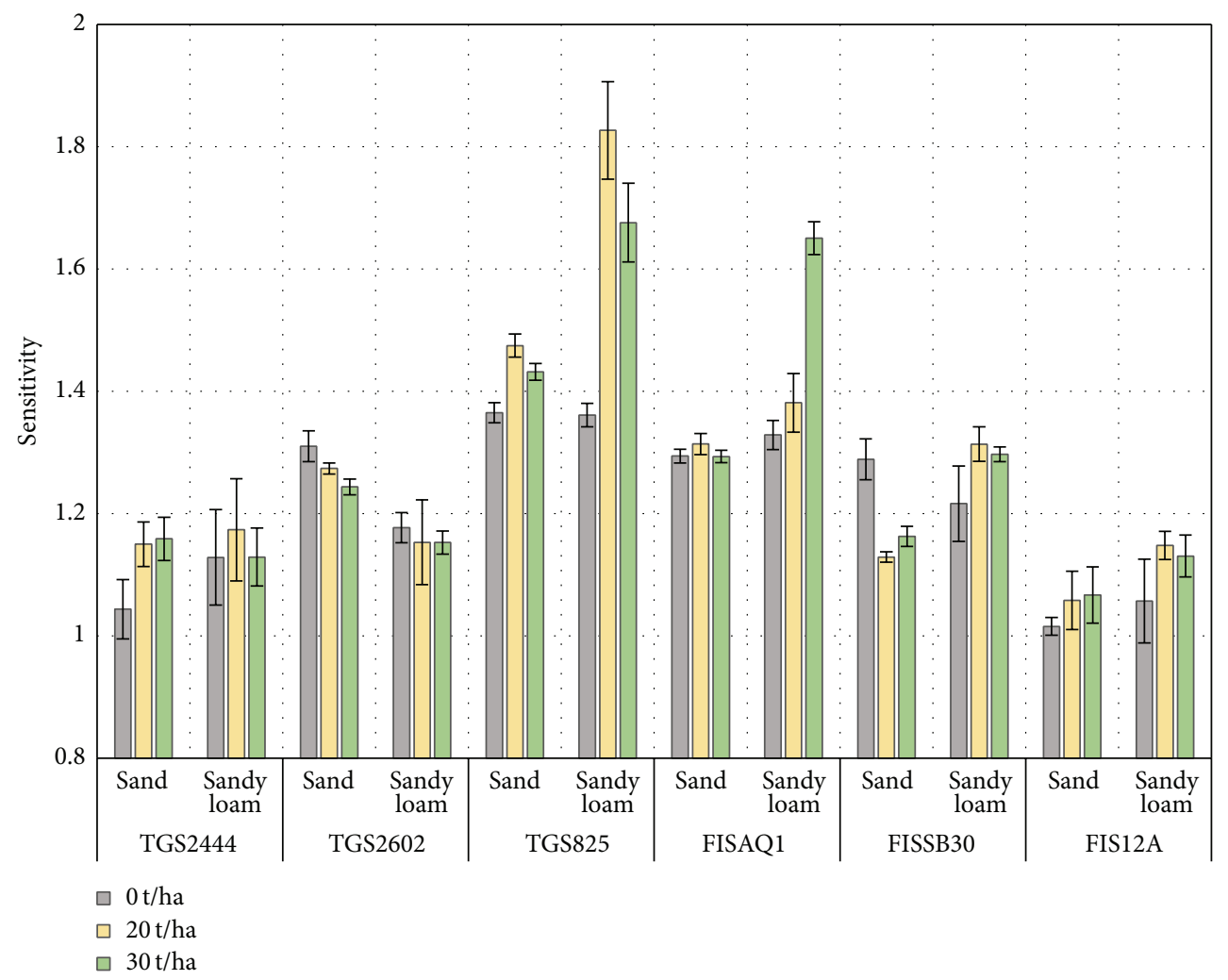

FIGURE 6: Individual sensitivity of sensor, the average and standard deviation of 5 replicates, to 3 levels of compost addition in different soil.

the amounts and types of soil gaseous compounds produced and released inside the $\mathrm{SH}$ atmosphere which corresponded to the soil type and doses of nutrient addition. Moreover, Figure 6 also presents the standard deviation of the MOS sensors to five replicates of each measurement. It relatively shows the low variance among responses which indicates the sufficient consistency of sensors reproducibility in producing the soil gaseous profiles on the same environment treatment independently throughout this study.

As shown in Figure 6, for most of the MOS gas sensors but TGS2602, the sensitivity to the nutrient addition (20 t/ha and $30 \mathrm{t} / \mathrm{ha}$ ) was higher than without nutrient addition whether for the same soil type or between sandy loam and sand. Sandy loam soil usually has more holding capacity of water and nutrient, along with lower bulk density compared to sand soil, thus leading to having more organic matter content [31, 33] and microorganism [34]. In addition, the use of a flow system (usually employing a pump) in sample detection causes cooling of the sensor surface, reducing the high increment of temperature and humidity inside such sensor chamber (heat dissipation) [35], thus also influencing its response.

Interestingly on TGS825 which is technically designed to respond to the hydrogen sulfide $\left(\mathrm{H}_{2} \mathrm{~S}\right)$ [36], it had the highest sensitivity among the others for each soil type. It reveals that the $\mathrm{H}_{2} \mathrm{~S}$ concentration during the headspace process was high and it is seen that the presence of nutrient addition contributed significantly to $\mathrm{H}_{2} \mathrm{~S}$ accumulation in the headspace (Figure 7). The response indicates that there is much acid sulfate material in soil samples. This gas can be produced from the oxidation process of organic material 


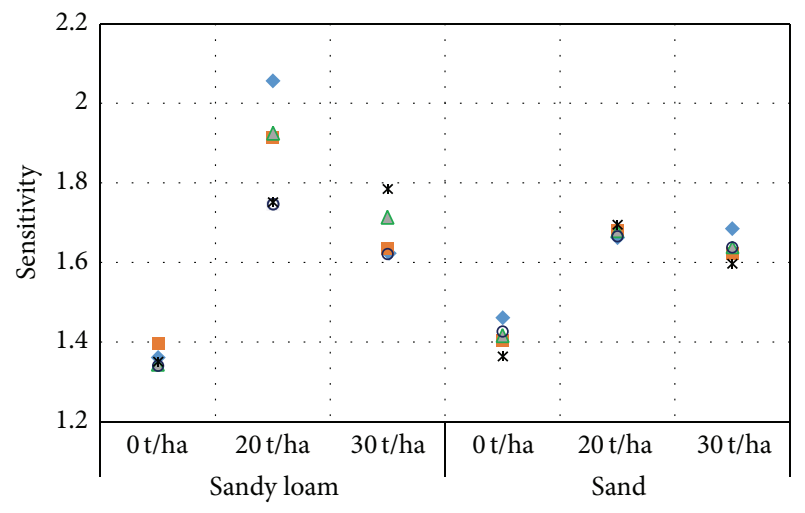

FIGURE 7: Response variances of TGS825 for five replicates between sandy loam and sand soil in different dose of nutrient addition.

containing sulfate acid due to bacterial activities in low oxygen environment (like flooded soil) which depends on ambient conditions such as temperature, humidity, and the concentration of certain metal ions [37]. The result also shows that the additional nutrient in sandy loam soil provided relatively higher concentration than in sand soil and there was a little cross-response in differentiating level of compost addition between doses $20 \mathrm{t} / \mathrm{ha}$ and $30 \mathrm{t} / \mathrm{ha}$.

The operation of temperature modulation-SDP through oscillating the heater voltage by square modulation does not only cause altering the kinetics of both adsorption and reaction process at the surface of sensor (effect of the frequency) but also consequently lead the MOS gas sensor to run at lower effective temperature (effect of the duty cycle), as on the TGS2444 which is driven by low duty cycle modulation [20] and shown to have high selectivity to ammonia gas [12]. For particular material, the specific working temperature provides optimum sensitivity for sensing a certain gas evidently [38, 39]. Ou et al. [39] found that under the low working temperature (i.e., $120^{\circ} \mathrm{C}$ ) a $2 \mathrm{D}$ metal disulfide-based gas sensor has very high selectivity to $\mathrm{NO}_{2}$ in which the sensing mechanism is dominated by charge transfer, adsorption between the surface-adsorbed $\mathrm{NO}_{2}$ gas molecules and metal disulfide, strongly due to paramagnetic behavior of $\mathrm{NO}_{2}$. Thus, the combination of frequency and effective working voltage by duty cycle selection of temperature modulationSDP had potential to sense sensitively the complex gas and/or volatile compounds of soil which then provide the unique gaseous profiles.

However, like typical characteristic of the use of sensor array in e-nose which does not allow individual sensor to identify a specific or complex volatile compounds, we found that there was no single sensor used which individually showed a relation for characterization of the difference of soil conditions clearly and linearly with regard to soil type and nutrient addition. There was a cross-response on each sensor in differentiating the dose level of nutrient addition, especially between normal dose ( $20 \mathrm{t} / \mathrm{ha}$ ) and high dose (30 t/ha). The complexity of soil gaseous compounds in potentially various kinds of gases, especially volatile compounds [16, 17], causes an inevitable cross-response on MOS gas sensor as also

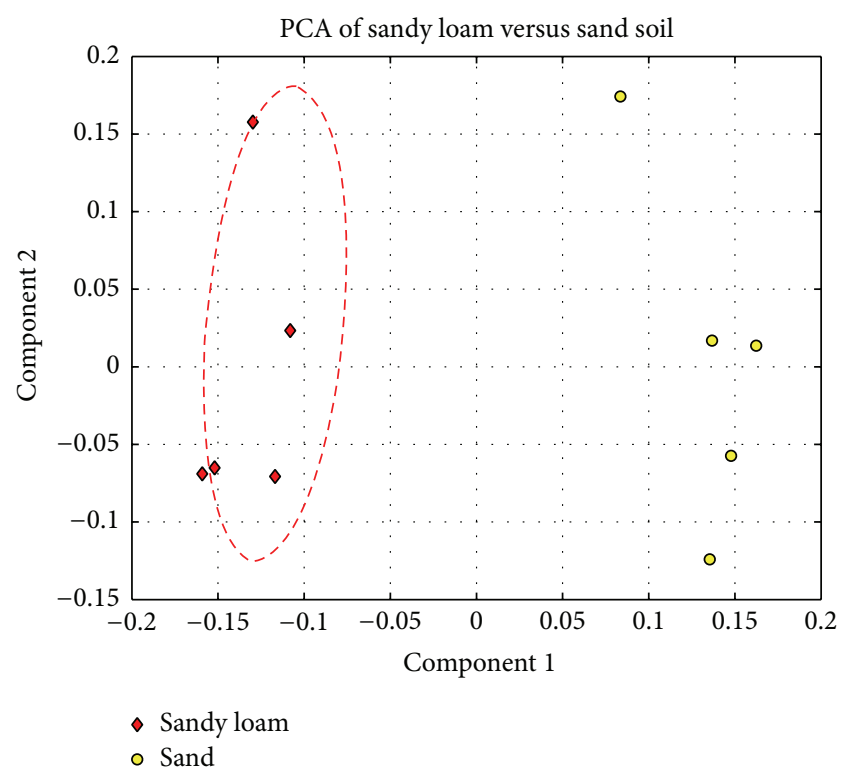

FIGURE 8: PCA plot showing discrimination between 2 soils without nutrient addition.

founded by Rincón et al. [40] who simulated a monitoring of VOC as soil contaminants through measuring 8 kinds of gases. The cross-response of individual sensor may be reduced by projecting collectively into new dimension using PCA as commonly used in e-nose.

\subsection{Performance of Discrimination of Soil under Different} Nutrient Addition. The potential of nonparametric biological system for discriminating soil type as well as for differentiating between different nutrient additions treatments based on its gaseous profile was tested. Firstly, the PCA, as a nonsupervised technique, was employed to find general relationships between samples while preserving most of the variance within data. PCA allow projecting variables onto fewer dimensions reflecting the most relevant analytical information [41]. This offers an advantage that the classification of unknowns is processed much faster, thus reducing detection time.

Figure 8 shows the PCA plot of discrimination of two soils, both without addition of compost. It shows a distinct zone of patterns volatile production between sandy loam soil and sand soil, where the principal component- (PC-) 1 accounts for higher differentiation of cluster than PC-2. PC1 and PC-2 cumulatively account for $78.32 \%$ of the variance within the data set.

Meanwhile, Figure 9 shows the PCA plot for replicates of each soil sample in distinguishing three doses of compost addition. It seem that PCA allow discriminating distinctly between soil conditions whether with or without compost (nutrient) addition, indicated by separated blue zone, even when differentiating regardless of soil type (Figure 9(c)).

It was only for sandy loam soil (Figure 9(a)); the level of compost addition could be clustered clearly into three groups as predefined previously, while there was misidentification between soils with dose $20 \mathrm{t} / \mathrm{ha}$ and dose $30 \mathrm{t} / \mathrm{ha}$ 


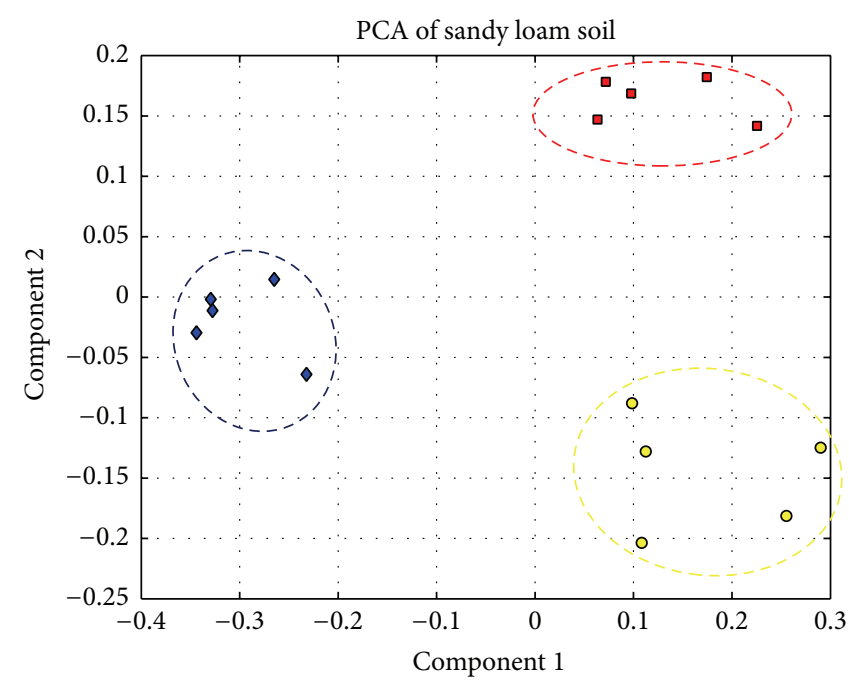

- No compost

- Compost $20 \mathrm{t}$

- Compost $30 \mathrm{t}$

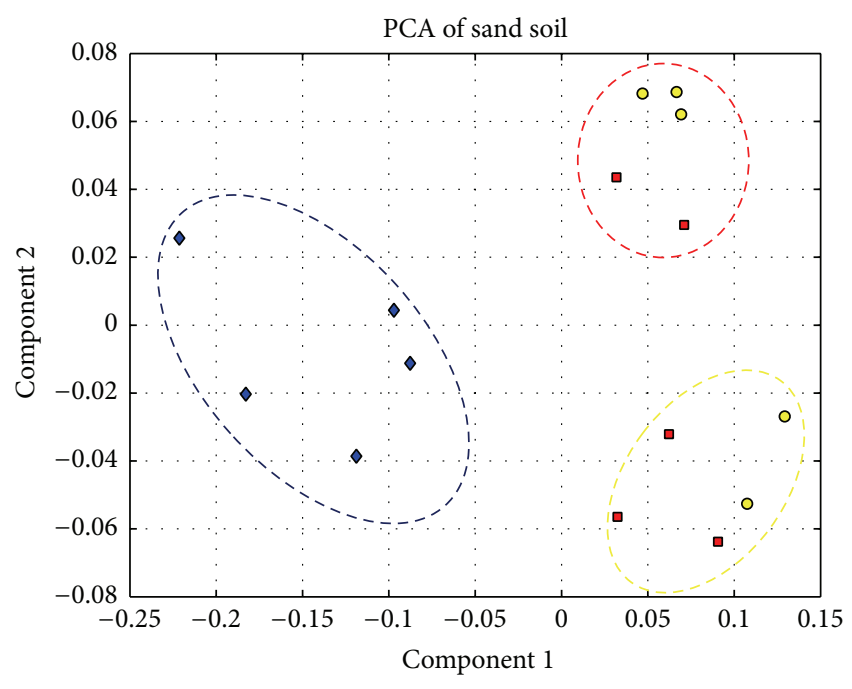

- No compost

- Compost $20 \mathrm{t}$

- Compost $30 \mathrm{t}$

(a)

(b)

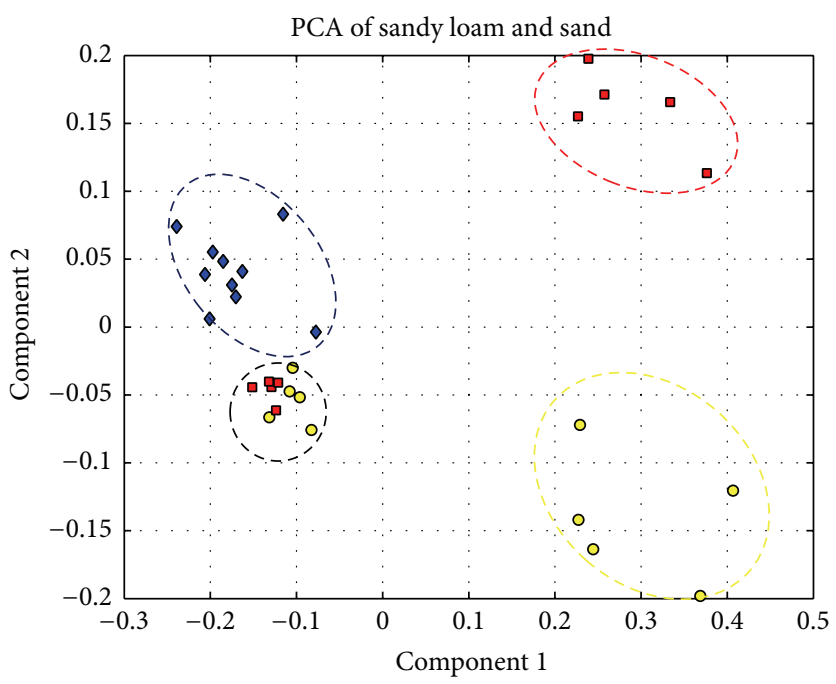

- No compost

- Compost $20 \mathrm{t}$

- Compost $30 \mathrm{t}$

(c)

FIGURE 9: PCA map for replicates of soil gaseous pattern projection for each soil sample in distinguishing three doses of compost addition (a) on sandy loam soil, (b) on sand soil, and (c) irrespective of soil type.

in sand soil (Figure 9(b)). Interestingly irrespective of soil type (Figure 9(c)), it seems to perform better in clustering the soil in different doses, yet there is a half part of replicates that has no clear classification (black zone) when identifying soil with doses $20 \mathrm{t} / \mathrm{ha}$ and $30 \mathrm{t} / \mathrm{ha}$. Figure 9 shows that the significant discrimination on the clusters between the soil without nutrient addition (blue zone) and soil with nutrient addition (yellow and red zones) was along the PC-1, while that between normal dose (yellow zone) and high dose (red zone) was mainly along the PC-2.
Finally, we determined the performance of NN as decision unit of e-nose to classify the level of nutrient addition in soil based on indicator the Mean Square Error (MSE) achieved resulting from the training process. We put three principal components (PCs) to distinguish the volatile compounds in the headspace released from soil samples as the input of neural network since they represent more than $90 \%$ of divergence samples data (Table 3). We designed the architecture of MLPNN that comprises 3 layers (single hidden layer). We determined the optimum number of neurons 
TABLE 3: Cumulative proportion of 3 PCs resulting from 6 sensors used.

\begin{tabular}{lccc}
\hline \multirow{2}{*}{ PC } & \multicolumn{3}{c}{ PCs proportion } \\
& Sandy loam & Sand & Irrespective of soil type \\
\hline PC-1 & $64.27 \%$ & $75.61 \%$ & $66.53 \%$ \\
PC-2 & $86.34 \%$ & $88.96 \%$ & $80.69 \%$ \\
PC-3 & $93.73 \%$ & $93.73 \%$ & $89.18 \%$ \\
\hline
\end{tabular}

TABle 4: Target definition for learning the soil gaseous patterns.

\begin{tabular}{lccl}
\hline T2 & T1 & T0 & Cluster category \\
\hline 0 & 0 & 1 & Soil without addition of compost \\
0 & 1 & 0 & Soil with compost doses of $20 \mathrm{t} / \mathrm{ha}$ \\
1 & 0 & 0 & Soil with compost doses of $30 \mathrm{t} / \mathrm{ha}$ \\
\hline
\end{tabular}

TABLE 5: MSE achieved by 6 neurons of hidden layer to discriminate 3 levels of compost addition in soil.

\begin{tabular}{lcc}
\hline Soil type & MSE of with PCA & MSE of without PCA \\
\hline Sand & $4.204 e-04$ & $3.490 e-03$ \\
Sandy loam & $1.226 e-04$ & $5.024 e-04$ \\
Regardless of type & $2.678 e-03$ & $4.080 e-03$ \\
\hline
\end{tabular}

in hidden layer by Singular Value Decomposition (SVD) analysis of its output in each training dataset [42]. By input from 3 PCs and based on the SVD value obtained, we choose 6 neurons in hidden layer to differentiate between the predescribed three categorized fertilizer levels in soil sample; thus, the neuron number architecture of MLPNN is 3-6-3 of, respectively, input, hidden, and output layer.

In learning, we took the learning parameters of $\mathrm{BP}$ as follow: maximum epoch is $10^{4}$, error target is $10^{-5}$, initial learning rate is 0.8 , and the constant of search time in searchthen-converge annealing learning rate is 700 . The target of output layer was defined as shown in Table 4 . We also trained the NN by input directly from sensors output (without preprocessing/PCA) with the same hidden layer (6-6-3 NN architecture). The achieved MSE of training results (Table 5) show that PCA helps in improving the NN classification to discriminate the level of compost addition in soil. In addition, all the application of trained data was successful to discriminate three levels of nutrient addition in soil.

The e-nose approach with static headspace method was potential for the aims of this work, providing different soil volatile profiles and allowing a discrimination between soil type and among the several soil treatments, to be obtained. This supports previous study where the same sampling method was employed for sensing the headspace of a soil under different condition and nutrient addition [15, 43], which may overcome the overlapping between volatile profiles. Compared with the results of Bastos and Magan [43], it seems that the use of sensors that potentially can detect gases/volatile compounds in complex compound provides better detection and economical value due to the small number of sensors used and the less complexity of the pattern identification system applied, rather than nonspecific sensors.

\section{Conclusions and Future Work}

The 6 selected MOS gas sensors with temperature modulation-SDP in e-nose system were promising, applied for indicating the presence of additional nutrients in soil since they could respond and have different sensitivity according to the samples. They provided (unique) soil gaseous profiles which accumulated in a static headspace optimized by thermostatting $\left(60^{\circ} \mathrm{C}\right)$ and stirring $(200 \mathrm{rpm})$ in controlled environment condition. The profiles show that the temperature modulation-SDP leads to distinguishing of the soils clearly and to indicating the presence of nutrient addition in soil. The MLPNN in single hidden layer architecture (3-6-3) with PCA as prior data preprocessor performed optimum identification in this study. The gas sensors with this particular technique offer a potential for replacing existing techniques in soil environmental fields for a quick and in situ application. It also suggests that it together with e-nose method could be used for monitoring microbial activity in soil and water as well. Depending on the applications and the type of sample to be analyzed, the choice of sensor array can be crucial for the good performance of the system.

\section{Competing Interests}

The authors declare that there are no competing interests regarding the publication of this paper.

\section{Acknowledgments}

Arief Sudarmaji is supported by Indonesian Directorate General of Higher Education (DIKTI) with Guarantee Letter no. 672/E4.4/K/2012 and Akio Kitagawa is supported by Japan Society for the Promotion of Science (JSPS) KAKENHI Grant nos. 25286036 and 15K12504.

\section{References}

[1] A. P. Lee and B. J. Reedy, “Temperature modulation in semiconductor gas sensing," Sensors and Actuators B: Chemical, vol. 60, no. 1, pp. 35-42, 1999.

[2] R. Chutia and M. Bhuyan, "Study of temperature modulated tin oxide gas sensor and identification of chemicals," in Proceedings of the 2nd National Conference on Computational Intelligence and Signal Processing (CISP '12), pp. 181-184, Guwahati, India, March 2012.

[3] X. Huang, F. Meng, Z. Pi, W. Xu, and J. Liu, "Gas sensing behavior of a single tin dioxide sensor under dynamic temperature modulation," Sensors and Actuators, B: Chemical, vol. 99, no. 23, pp. 444-450, 2004.

[4] X. Huang, J. Liu, D. Shao, Z. Pi, and Z. Yu, "Rectangular mode of operation for detecting pesticide residue by using a single $\mathrm{SnO}_{2}$ based gas sensor," Sensors and Actuators B: Chemical, vol. 96, no. 3, pp. 630-635, 2003.

[5] E. Martinelli, D. Polese, A. Catini, A. D’Amico, and C. Di Natale, "Self-adapted temperature modulation in metal-oxide semiconductor gas sensors," Sensors and Actuators B: Chemical, vol. 161, no. 1, pp. 534-541, 2012.

[6] A. Vergara, E. Martinelli, E. Llobet, A. D’amico, and C. Di Natale, "Optimized feature extraction for temperature-modulated gas 
sensors," Journal of Sensors, vol. 2009, Article ID 716316, 10 pages, 2009.

[7] E. Brauns, E. Morsbach, S. Kunz, M. Baeumer, and W. Lang, "Temperature modulation of a catalytic gas sensor," Sensors (Switzerland), vol. 14, no. 11, pp. 20372-20381, 2014.

[8] S. Nakata and K. Kashima, "Distinguishing among gases with a semiconductor sensor depending on the frequency modulation of a cyclic temperature," Electroanalysis, vol. 22, no. 14, pp. 15731580, 2010.

[9] S. Nakata, H. Okunishi, and Y. Nakashima, "Distinction of gases with a semiconductor sensor under a cyclic temperature modulation with second-harmonic heating," Sensors and Actuators B: Chemical, vol. 119, no. 2, pp. 556-561, 2006.

[10] K. A. Ngo, P. Lauque, and K. Aguir, "High performance of a gas identification system using sensor array and temperature modulation," Sensors and Actuators, B: Chemical, vol. 124, no. 1, pp. 209-216, 2007.

[11] A. Fort, M. Gregorkiewitz, N. Machetti et al., "Selectivity enhancement of $\mathrm{SnO}_{2}$ sensors by means of operating temperature modulation," Thin Solid Films, vol. 418, no. 1, pp. 2-8, 2002.

[12] A. Sudarmaji and A. Kitagawa, "Sensors \& transducers temperature modulation with specified detection point on metal oxide semiconductor gas sensors for E-nose application," Sensors \& Transducers, vol. 186, no. 3, pp. 93-103, 2015.

[13] T. Carson, C. M. Bachmann, and C. Salvaggio, "Soil signature simulation of complex mixtures and particle size distributions," Optical Engineering, vol. 54, no. 9, Article ID 094103, 2015.

[14] Soil Science Society of America, "Soils-Overview," Water Resources, 2010, https://www.soils.org/files/about-soils/soilsoverview.pdf.

[15] F. De Cesare, E. Di Mattia, S. Pantalei et al., "Use of electronic nose technology to measure soil microbial activity through biogenic volatile organic compounds and gases release," Soil Biology and Biochemistry, vol. 43, no. 10, pp. 2094-2107, 2011.

[16] H. Insam and M. S. A. Seewald, "Volatile organic compounds (VOCs) in soils," Biology and Fertility of Soils, vol. 46, no. 3, pp. 199-213, 2010.

[17] F. Tassi, S. Venturi, J. Cabassi, F. Capecchiacci, B. Nisi, and O. Vaselli, "Volatile organic compounds (VOCs) in soil gases from Solfatara crater (Campi Flegrei, southern Italy): geogenic source(s) vs. biogeochemical processes," Applied Geochemistry, vol. 56, pp. 37-49, 2015.

[18] C. Mei Wang and D. E. Cane, "NIH public access," Journal of the American Chemical Society, vol. 29, no. 6, pp. 997-1003, 2008.

[19] C.-M. Wang and D. E. Cane, "Biochemistry and molecular genetics of the biosynthesis of the earthy odorant methylisoborneol in Streptomyces coelicolor," Journal of the American Chemical Society, vol. 130, no. 28, pp. 8908-8909, 2008.

[20] Figaro Engineering Inc, Data Sheet TGS 2444 for the Detection of Ammonia, 2011.

[21] D. Hercog and B. Gergič, "A flexible microcontroller-based data acquisition device," Sensors, vol. 14, no. 6, pp. 9755-9775, 2014.

[22] M. A. Naivar, M. E. Wilder, R. C. Habbersett et al., "Development of small and inexpensive digital data acquisition systems using a microcontroller-based approach," Cytometry Part A, vol. 75, no. 12, pp. 979-989, 2009.

[23] R. Gutierrez-Osuna, H. T. Nagle, B. Kermani, and S. S. Schiffman, "Introduction to chemosensors," in Handbook of Machine Olfaction, T. C. Pearce, S. S. Schiffman, H. T. Nagle, and J. W. Gardner, Eds., pp. 133-160, Wiley-VCH Verlag GmbH \& Co. KGaA, Weinheim, Germany, 2003.
[24] A. Sudarmaji, A. Kitagawa, and J. Akita, "Design of wireless measurement of soil gases and soil environment based on Programmable System-on-Chip (PSOC)," in Proceedings of the International Symposium on Agricultural and Biosystem Engineering (ISABE '13), pp. E5-1-E5-13, 2013.

[25] K.-L. Du and M. N. S. Swamy, Neural Networks and Statistical Learning, Springer, London, UK, 2014.

[26] N. Haber, B. Deller, H. Flaig, E. Schulz, and J. Reinhold, "Sustainable compost application in agriculture," ECN-INFO 02/2010, European Compost Network, 2008.

[27] A. R. Conklin, Introduction to Soil Chemistry: Analysis and Instrumentation, John Wiley \& Sons, Hoboken, NJ, USA, 2nd edition, 2014.

[28] K. Malone and H. Williams, Growing Season Definition and Use in Wetland Delineation: A Literature Review, US Army Engineer Research and Development Center, Nacogdoches, Tex, USA, 2010.

[29] M. C. Rabenhorst, "Biologic zero: a soil temperature concept," Wetlands, vol. 25, no. 3, pp. 616-621, 2005.

[30] C. Yu, J. Cheng, L. Jones et al., "Data collection handbook to support modeling the impacts of radioactive material in soil," Tech. Rep., Argonne National Laboratory, Argonne, Ill, USA, 1993.

[31] P. R. Chaudhari, D. V. Ahire, V. D. Ahire, M. Chkravarty, and S. Maity, "Soil bulk density as related to soil texture, organic matter content and available total nutrients of Coimbatore soil," International Journal of Scientific and Research Publications, vol. 3, no. 2, pp. 1-8, 2013.

[32] Corning, Instruction Manual: For All Hot Plates, Stirrers, and Stirrer/Hot Plates with Digital Displays and for the 6795PR Temperature Controller, Corning, Lowell, Mass, USA, 2007.

[33] J. A. Amador and J. A. Atoyan, "Structure and composition of leachfield bacterial communities: role of soil texture, depth and septic tank effluent inputs," Water, vol. 4, no. 3, pp. 707-719, 2012.

[34] N. H. Hamarashid, M. A. Othman, and M.-A. H. Hussain, "Effects of soil texture on chemical compositions, microbial populations and carbon mineralization in soil," The Egyptian Journal of Experimental Biology, vol. 6, no. 1, pp. 59-64, 2010.

[35] Figaro Engineering Inc, General Information for TGS Sensors: Technical Information on Usage of TGS Sensors for Toxic and Explosive Gas Leak Detectors, Figaro Engineering Inc, 2005.

[36] Figaro Engineering Inc, Product Information TGS 825-Special Sensor for Hydrogen Sulfide, 2011.

[37] S. Chou, J. M. Ogden, H. R. Pohl et al., Draft Toxicological Profile for Hydrogen Sulfide and Carboxyl Sulfide, Agency for Toxic Substances and Disease Registry, Atlanta, Ga, USA, 2014.

[38] D. N. Chavan, G. E. Patil, D. D. Kajale, V. B. Gaikwad, P. K. Khanna, and G. H. Jain, "Nano Ag-doped $\operatorname{In}_{2} \mathrm{O}_{3}$ thick film: a low-temperature $\mathrm{H}_{2} \mathrm{~S}$ gas sensor," Journal of Sensors, vol. 2011, Article ID 824215, 8 pages, 2011.

[39] J. Z. Ou, W. Ge, B. Carey et al., "Physisorption-based charge transfer in two-dimensional $\mathrm{SnS}_{2}$ for selective and reversible $\mathrm{NO}_{2}$ gas sensing," ACS Nano, vol. 9, no. 10, pp. 10313-10323, 2015.

[40] M. Rincón, J. M. Getino, J. Robla, G. Hierro, J. Mochón, and I. Bustinza, "Gas sensor array for VOC's monitoring in soils contamination," Ingeniería, vol. 14, no. 1, pp. 45-54, 2010.

[41] E. L. Hines, P. Boilot, J. W. Gardner, and M. A. Gongora, "Pattern analysis for electronic noses," in Handbook of Machine Olfaction: Electronic Nose Technology, T. C. Pearce, S. S. Schiffman, 
H. T. Nagle, and J. W. Gardner, Eds., chapter 6, pp. 133-160, WILEY-VCH, Weinheim, Germany, 2003.

[42] J. D. A. Santos, G. A. Barreto, and C. M. S. Medeiros, "Estimating the number of hidden neurons of the MLP using singular value decomposition and principal components analysis: a novel approach," in Proceedings of the 11th Brazilian Symposium on Neural Networks (SBRN '10), pp. 19-24, IEEE, Sao Paulo, Brazil, October 2010.

[43] A. C. Bastos and N. Magan, "Soil volatile fingerprints: use for discrimination between soil types under different environmental conditions," Sensors and Actuators B: Chemical, vol. 125, no. 2, pp. 556-562, 2007.

[44] Figaro Engineering Inc, TGS 2602-For the Detection of Air Contaminants, 2005.

[45] FIS Inc, FIS GAS SENSOR SB-12A for Methane Detection, 2006.

[46] FIS, FIS Gas Sensor SB-30 for Alcohol Detection, FIS, 2008.

[47] FIS Inc, FIS Gas Sensor SB-AQ1 for Air Quality Control (VOCs), 2010. 


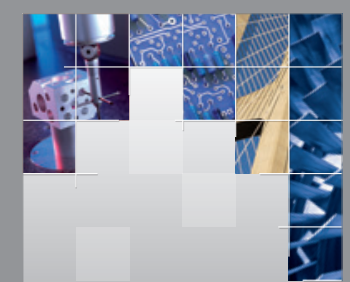

\section{Enfincering}
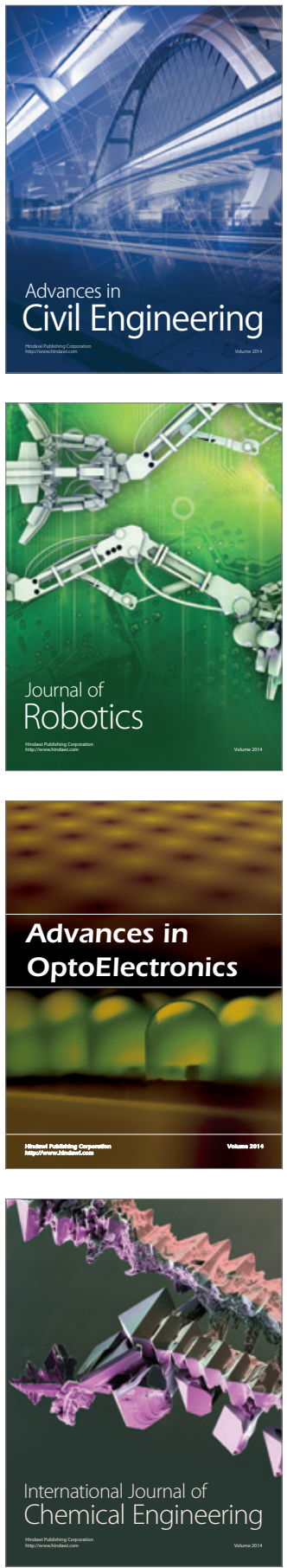

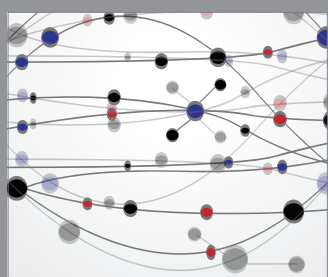

The Scientific World Journal

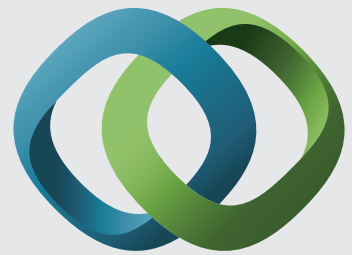

\section{Hindawi}

Submit your manuscripts at

http://www.hindawi.com
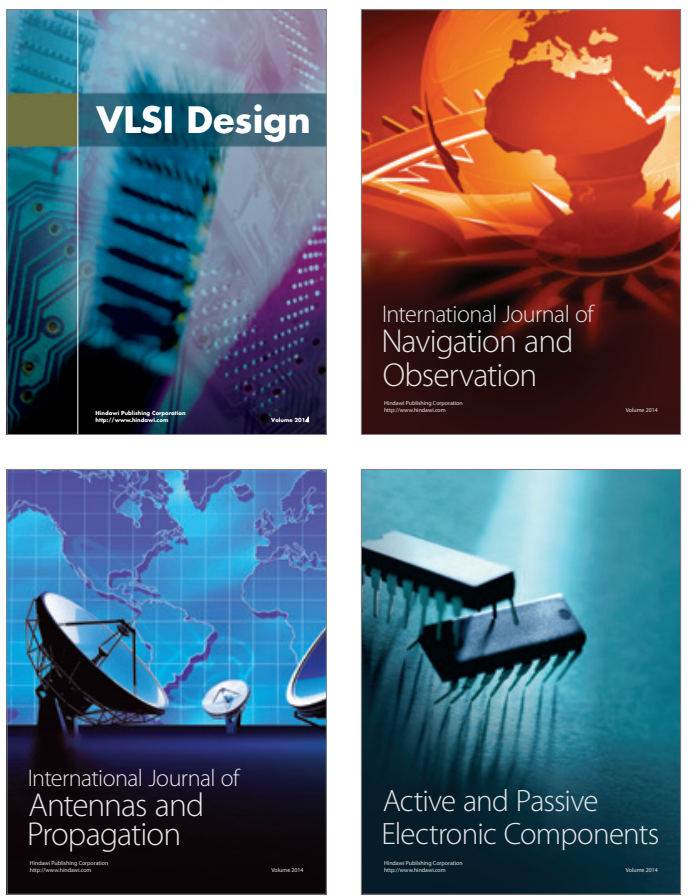
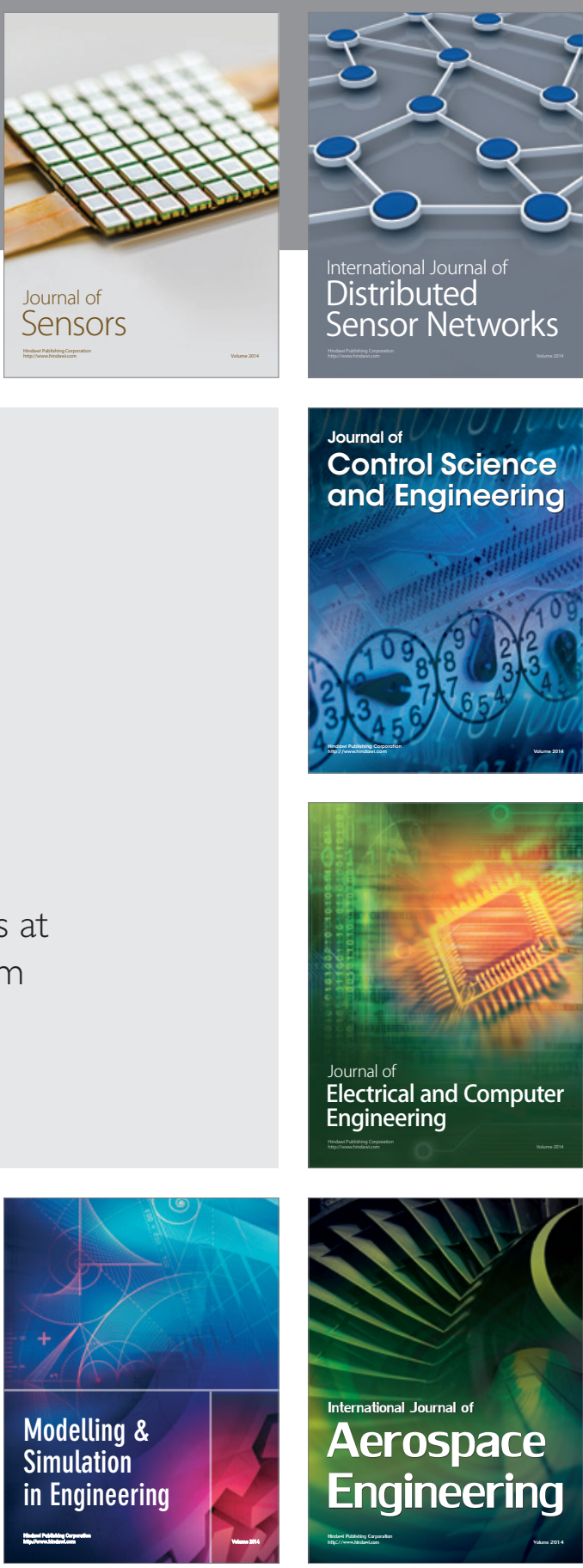

International Journal of

Distributed

Sensor Networks

Journal of

Control Science

and Engineering
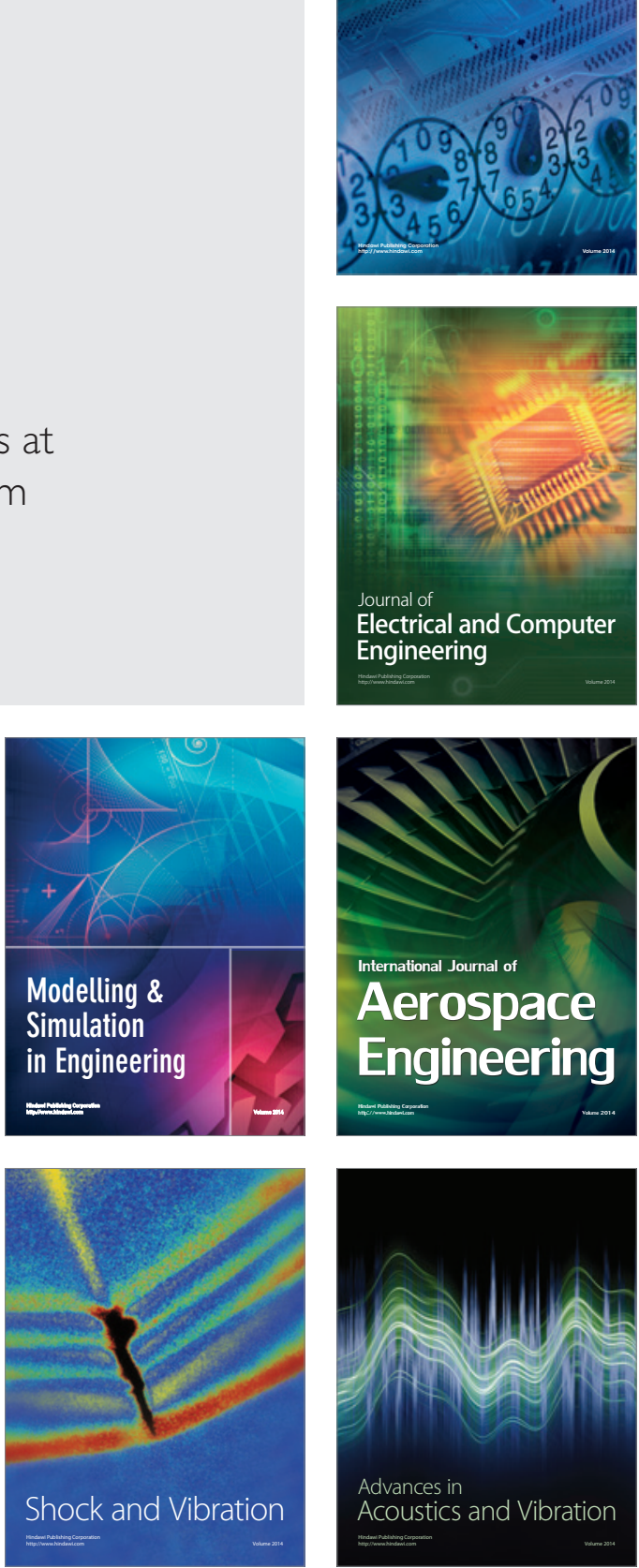